Addendum to the van der Whal s const ant s anong ground- st at e hydrogen at ons

\begin{tabular}{|l|l|}
\hline 著者 & KOGA Toshi kat su \\
\hline $\begin{array}{l}\text { j our nal or } \\
\text { publ i cat } \mathrm{i} \text { on } \mathrm{titl} \text { e }\end{array}$ & The $\mathrm{j}$ our nal of chemi cal physics \\
\hline vol une & 90 \\
\hline number & 1 \\
\hline page range & $605-606$ \\
\hline year & $1989-01-01$ \\
\hline URL & ht t p: //hdl . handl e. net $/ 10258 / 864$ \\
\hline
\end{tabular}




\title{
Addendum to the van der Waals constants among ground-state hydrogen atoms
}

\author{
Toshikatsu Koga \\ Department of Applied Chemistry and Department of Applied Science for Energy, \\ Muroran Institute of Technology, Muroran, Hokkaido 050, Japan
}

(Received 11 July 1988; accepted 15 September 1988)

In a series of papers ${ }^{1-6}$ published in this Journal, a momentum-space method has been developed and applied to the problem of van der Waals interaction between two ground-state hydrogen atoms. The Fock transformation ${ }^{7-9}$ of the momentum variable has been employed to expand the integral kernel of the momentum-space perturbation equation in terms of the four-dimensional spherical harmonics. Then the perturbation equation is reduced to an infinite number of algebraic linear equations, whose solution with an appropriate truncation yields the coefficients of the linear combination of spherical harmonics used to represent the perturbation wave function. High accuracy of the resultant nonvariational wave function in a series form has been verified ${ }^{10}$ based on the local energy ${ }^{11,12}$ examination in both momentum and position spaces. Succeedingly, these accurate perturbation wave functions have been used ${ }^{1-6}$ to evaluate various van der Waals constants between ground-state hydrogen atoms, including the leading relativistic correction $W_{4}$.

In the present note, we first show the fundamental significance of the two- and three-body interactions in a general $N$-hydrogen atomic system. We then report some van der Waals constants for the nonadditive three-body interaction, obtained directly from the above perturbation wave function. We also report improved values for several two-body interaction constants; a few of their previous values ${ }^{2}$ have been found to be incorrect in the last two decimal places.

Let us consider the van der Waals interaction system of $N$ ground-state hydrogen atoms. Since the perturbation potential is the sum of pairwise potentials, the first order perturbation wave function of this system can be expressed as

$\Psi^{(1)}(1,2, \ldots, N)=\sum_{i>j=1}^{N}\left[\psi^{(1)}(i, j) \sum_{k(\neq i, j)}^{N} 1 s(\dot{k} \nmid]\right.$

where $1 s(k)$ is the $1 s$ orbital on the $k$ th atom and $\psi^{(1)}(i, j)$ is the two-body first order perturbation wave function for a pair of atoms $i$ and $j$. Then the first order energy $E^{(1)}$ vanishes identically, while the second order energy $E^{(2)}$ is found to be purely additive:

$$
E^{(2)}=\sum_{i>j=1}^{N} \epsilon^{(2)}(i, j)
$$

$$
\epsilon^{(2)}(i, j)=\left\langle 1 s(i) 1 s(j)\left|V^{(1)}(i, j)\right| \psi^{(1)}(i, j)\right\rangle,
$$

where $\epsilon^{(2)}(i, j)$ is the second order energy for a pair of atoms $i$ and $j$ resulting from the perturbation potential $V^{(1)}(i, j)$. The third order energy $E^{(3)}$ consists of the additive two-body and nonadditive three-body terms:

$$
\begin{aligned}
& E^{(3)}=\sum_{i>j=1}^{N} \epsilon^{(3)}(i, j)+\sum_{i>j>k=1}^{N} \epsilon^{(3)}(i, j, k), \\
& \epsilon^{(3)}(i, j)=\left\langle\psi^{(1)}(i, j)\left|V^{(1)}(i, j)\right| \psi^{(1)}(i, j)\right\rangle, \\
& \epsilon^{(3)}(i, j, k) \\
& =2\left\langle\psi^{(1)}(i, j) 1 s(k)\left|V^{(1)}(k, i)\right| \psi^{(1)}(j, k) 1 s(i)\right\rangle \\
& \quad+2\left\langle\psi^{(1)}(j, k) 1 s(i)\left|V^{(1)}(i, j)\right| \psi^{(1)}(k, i) 1 s(j)\right\rangle \\
& \quad+2\left\langle\psi^{(1)}(k, i) 1 s(j)\left|V^{(1)}(j, k)\right| \psi^{(1)}(i, j) 1 s(k)\right\rangle,(3 \mathrm{c})
\end{aligned}
$$

where $\epsilon^{(3)}(i, j)$ and $\epsilon^{(3)}(i, j, k)$ are the third order two- and three-body energies, respectively. So long as we consider the perturbation correction up to the third order, the van der Waals interaction among $N$ hydrogen atoms can be described by the two-body energies $\epsilon^{(2)}(i, j)$ and $\epsilon^{(3)}(i, j)$ and the three-body energy $\epsilon^{(3)}(i, j, k)$. Thus the two- and threebody interaction constants are fundamental quantities for the van der Waals interaction.

When the multipole expansion ${ }^{13,14}$ is applied to the perturbation potential, the two-body function $\psi^{(1)}(i, j)$, and hence the interaction energy, are expanded as a power series of the reciprocal interatomic distance $R_{i j}^{-1}$. The procedure for obtaining the coefficients $C_{n}$ of $R_{i j}^{-n}(6 \leqslant n \leqslant 11)$ for the two-body energies $\epsilon^{(2)}(i, j)$ and $\epsilon^{(3)}(i, j)$ has been already described in detail. ${ }^{1,2,4}$

The evaluation of the three-body energy $\epsilon^{(3)}(i, j, k)$ requires an additional rotation of spherical harmonics, since the function $\psi^{(1)}(i, j)$ is given in a local coordinate system defined by the line connecting the atoms $i$ and $j$. This can be performed by the introduction of the rotational matrix, ${ }^{15,16}$ and each term of the three-body contribution is found ${ }^{17}$ to be a product of an interaction constant and a geometrical factor which depends on the relative conformation of the three atoms. The latter coincides with the expression given by 
TABLE I. Several van der Waals coefficients among ground-state hydrogen atoms in atomic units.

\begin{tabular}{|c|c|}
\hline Two-body interaction & Three-body interaction \\
\hline 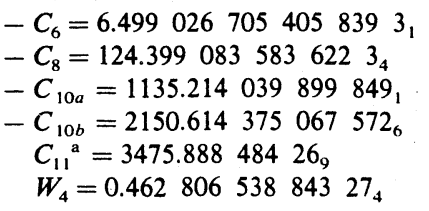 & 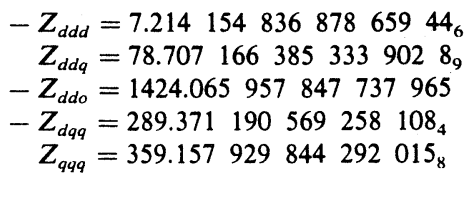 \\
\hline
\end{tabular}

a The leading third order term proportional to $R^{-11}$.

Bell. ${ }^{18}$ The interaction constants $Z_{l_{1} l_{2} l_{3}}$ are determined by the coefficients of four-dimensional spherical harmonics appearing in $\psi^{(1)}(i, j)$.

Using the perturbation wave function given in Refs. 1 and 2 , we have been able to evaluate these constants very accurately for five types of interactions, i.e., $d d d, d d q, d d o$, $d q q$, and $q q q$ interactions where $d, q$, and $o$ mean, respectively, the dipole, quadrupole, and octupole contributions. Our final values for the five types of interaction constants are summarized in Table I. We have obtained convergent values with a few more significant figures than Thakkar, ${ }^{19}$ who has very recently reported an accurate and extensive compilation of the van der Waals constants for the hydrogen atom based on the pseudostate method (see also Ref. 20). Also tabulated in Table I are several two-body interaction constants with improved accuracy. The previous values ${ }^{2}$ for $C_{8}$, $C_{10 a}$, and $C_{10 b}$ are found to be incorrect in their last two decimal places. The table is a summary of the van der Waals constants for the ground-state hydrogen atom, obtained $d i$ rectly from the perturbation wave function resulting from the nonvariational momentum-space method.

The author thanks Professor A. J. Thakkar for his comments on our previous values for $C_{8}, C_{10 a}$, and $C_{10 b}$, and for a preprint of Ref. 19. He is also grateful to Mr. T. Matsuhashi for his computational assistance.

'T. Koga and S. Matsumoto, J. Chem. Phys. 82, 5127 (1985).

${ }^{2}$ T. Koga and M. Uji-ie, J. Chem. Phys. 84, 335 (1986).

${ }^{3}$ T. Koga, J. Chem. Phys. 83, 6304 (1985).

${ }^{4}$ T. Koga and M. Uji-ie, J. Chem. Phys. 87, 1137 (1987).

${ }^{5}$ T. Koga, J. Chem. Phys. 84, 1636 (1986).

${ }^{6}$ T. Koga and M. Uji-ie, J. Chem. Phys. 86, 2854 (1987).

${ }^{7}$ V. Fock, Z. Phys. 98, 145 (1935).

${ }^{8}$ T. Shibuya and C. E. Wulfman, Proc. R. Soc. London Ser. A 286, 376 (1965).

${ }^{9}$ B. R. Judd, Angular Momentum Theory for Diatomic Molecules (Academic, New York, 1975).

${ }^{10}$ T. Koga, T. Uchiyama, and M. Uji-ie, J. Chem. Phys. 87, 4025 (1987).

"J. H. Bartlett, Phys. Rev. 51, 661 (1937).

${ }^{12}$ A. A. Frost, J. Chem. Phys. 10, 240 (1942); A. A. Frost, R. E. Kellog, and E. C. Curtis, Rev. Mod. Phys. 32, 313 (1960).

${ }^{13}$ J. S. Dahler and J. O. Hirschfelder, J. Chem. Phys. 25, 986 (1956).

${ }^{14} \mathrm{~J}$. O. Hirschfelder and P.-O. Löwdin, Mol. Phys. 2, 229 (1959).

${ }^{15}$ L. C. Biedenharn and J. D. Louck, Angular Momentum in Quantum Physics. Theory and Application (Addison-Wesley, Reading, 1981).

${ }^{16}$ M. E. Rose, Elementary Theory of Angular Momentum (Wiley, New York, 1957).

${ }^{17} \mathrm{~T}$. Koga and M. Uji-ie (unpublished).

${ }^{18}$ R. J. Bell, J. Phys. B 3, 751, L63 (1970).

${ }^{19}$ A. J. Thakker, J. Chem. Phys. 89, 2092 (1988).

${ }^{20}$ V. Magnasco and G. Figari, Mol. Phys. 62, 1419 (1987). 